\title{
ADSORÇÃO DE 3-(3,4-DICLOROFENIL)-1,1-DIMETILURÉIA (DIURON) UTILIZANDO PINHA DE Pinus elliottii
}

\author{
T. J. LOPES ${ }^{1}$, M. S. CAMARGO ${ }^{1}$, M. D. SCARDUELI ${ }^{1}$, N. L. SANTOS ${ }^{1}$, M. A. SANTOS ${ }^{1}$, M. B. \\ QUADRI $^{2}$, M. H. S. KURZ ${ }^{1}$ e F. F. GONÇALVES ${ }^{1}$ \\ ${ }^{1}$ Universidade Federal do Rio Grande, Escola de Química e Alimentos. \\ ${ }^{2}$ Universidade Federal de Santa Catarina, Departamento de Engenharia Química e Engenharia de \\ Alimentos \\ E-mail para contato: tjlopes@furg.br
}

\begin{abstract}
RESUMO - O presente trabalho objetivou a utilização de um material adsorvente alternativo, pinha de Pinus elliotti, como adsorvente de baixo custo para a remoção do herbicida Diuron de recursos hídricos. Os ensaios de adsorção foram conduzidos em sistema em batelada para avaliar a cinética e a isoterma de adsorção. Também, foi avaliada a influência de 3 fatores utilizando técnicas de planejamento experimental:

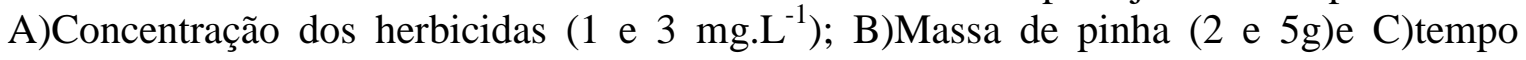
(50min e 2 horas). A concentração de Diuron foi determinada através da utilização de um HPLC-DAD com fase móvel acetonitrila:água pH 3,0 $(220 \mathrm{~nm})$. Os resultados obtidos demonstram que a pinha apresenta um melhor ajuste para isoterma de Freundlich (correlação de 0,9939), todos os fatores testados se mostraram significativos e foi obtida uma adsorção máxima de $80 \%$ da concentração inicial de herbicida. Portanto, a pinha se mostrou um adsorvente promissor para a adsorção do Diuron em meio aquoso.
\end{abstract}

\section{INTRODUÇÃO}

O Brasil é responsável pelo consumo de cerca de $50 \%$ da quantidade de agrotóxicos utilizados na América Latina, o que envolve um comércio estimado em cerca de US\$ 2.56 bilhões em 1998 (SINDAG, 2012).

A água é um dos constituintes essenciais do meio ambiente para a sobrevivência dos organismos vivos, fazendo parte dos ciclos biológicos, geológicos e químicos que mantém em equilíbrio os ecossistemas. Além, da manutenção da vida, a água tem uso primordial na agricultura, indústria, alimentação e recreação. No entanto, a má disposição final de agrotóxicos e efluentes, promovem um grande problema de agressão aos recursos hídricos, pois se estes não forem tratados e dispostos devidamente, causam uma série de problemas físicos, químicos e biológicos num corpo d'água (Porto, 1991).

Neste cenário, foi utilizado neste estudo, o herbicida DIURON que é indicado para as culturas de cana-de-açúcar, citros, café, algodão, entre outras. O diuron [3-(3,4-diclorofenil)-1,1-dimetiluréia], pertencente ao grupo químico das feniluréias (Musumeci et al., 1999). 
O impacto das águas contaminadas no meio ambiente depende da sua composição química, da quantidade e da velocidade com que estes são lançados no meio e da capacidade daquele ambiente natural em neutralizar os efeitos adversos causados pelos poluentes (Soares, 2005). Existem vários procedimentos disponíveis para a remoção de agrotóxicos de água: degradação fotocatalítica (Gong et al., 2011), processos oxidativos avançados (Zhou et al., 2011), tratamentos biológicos (Katayama e Matsumura, 1993) e adsorção (Santos et al., 2013; Al-Muhtase et al., 2011).

A adsorção é um dos métodos mais eficientes empregados na remoção de cor, odor, óleos e poluentes orgânicos. Muitos materiais de baixo custo têm sido estudados devido às suas vantagens econômicas. Dentre esses, podemos citar: madeira, casca de laranja, bagaço de cana-de-açúcar, argilas, sílica, casca de amendoim, bambu etc (Namasivayam et al., 1996).

O presente trabalho teve como objetivo o estudo da utilização de um material adsorvente alternativo, pinha de Pinus elliotti, como adsorvente de baixo custo para a remoção de Diuron de recursos hídricos.

\section{MATERIAL E MÉTODOS}

Os experimentos em sistema estático (batelada) foram conduzidos a $20^{\circ} \mathrm{C}$, agitação de $150 \mathrm{rpm}$, com 40 mL de solução de Diuron de concentração inicial $\left(0,3\right.$ a 3,7 mg. $\left.\mathrm{L}^{-1}\right)$ e foi adicionada massa de pinha conhecidas. Após determinados períodos de tempo, foram retiradas alíquotas do sobrenadante para realizar a determinação da concentração de herbicida em um HPLC-DAD com fase móvel acetonitrila:água pH 3,0 e um comprimento de onda de $220 \mathrm{~nm}$.

\subsection{Isotermas e Cinética de Adsorção}

O conjunto de valores experimentais obtidos procurou-se descrever através de modelos de mecanismos simultâneos de difusão no interior da partícula e de convecção na superfície externa da mesma. A Equação 1 representa o balanço diferencial de massa na partícula incluindo a parcela de adsorção sobre a fase sólida, dada pelo segundo termo do membro esquerdo.

$$
\varepsilon_{\mathrm{p}} \frac{\partial \mathrm{C}_{\mathrm{i}}}{\partial \mathrm{t}}+\rho_{\mathrm{d}} \frac{\partial \mathrm{q}_{\mathrm{i}}}{\partial \mathrm{t}}=\mathrm{D}_{\text {ef }} \varepsilon_{\mathrm{p}}\left(\frac{\partial^{2} \mathrm{C}_{\mathrm{i}}}{\partial \mathrm{r}^{2}}+\frac{2}{\mathrm{r}} \frac{\partial \mathrm{C}_{\mathrm{i}}}{\partial \mathrm{r}}\right)
$$

Condição inicial: $\mathrm{t}=0$;

$$
\mathrm{Ci}=0 ; q \mathrm{i}=0
$$

Condição de contorno:

No centro da partícula: $\quad \mathrm{r}=0$;

$$
\frac{\partial \mathrm{Ci}_{\mathrm{i}}}{\partial \mathrm{r}}=0
$$


Na superfície externa: $\quad \mathrm{r}=\mathrm{R}$;

$$
\frac{\partial \mathrm{C}_{\mathrm{i}}}{\partial \mathrm{r}}=\frac{\mathrm{k}_{\text {conv }}}{\text { Def }}\left(\mathrm{C}^{\infty}(\mathrm{t})-\mathrm{C}_{\mathrm{i}}\right)
$$

Onde $\varepsilon p=$ porosidade da partícula; $\mathrm{C}_{\mathrm{i}}=$ concentração de herbicida na fase líquida no interior dos poros da partícula $\left(\mathrm{mg} . \mathrm{cm}^{-3}\right) ; \rho d=$ massa específica da partícula $\left(\mathrm{g} . \mathrm{cm}^{-3}\right) ; \mathrm{q}_{\mathrm{i}}=$ concentração de herbicida na fase sólida $\left(\mathrm{mg} \cdot \mathrm{g}^{-1}\right) ; \mathrm{D}_{\mathrm{ef}}=$ coeficiente de difusão efetivo $\left(\mathrm{cm}^{2} \cdot \mathrm{s}^{-1}\right) ; \mathrm{t}=$ tempo $(\mathrm{s})$; $\mathrm{r}=$ coordenada radial $(\mathrm{cm}) ; \mathrm{C}^{*}=$ concentração de herbicida na fase líquida no interior dos poros da partícula em equilíbrio ao final de um processo de adsorção $\left(\mathrm{mg} . \mathrm{cm}^{-3}\right) ; \mathrm{R}=$ raio da partícula $(\mathrm{cm})$; $\mathrm{k}_{\text {conv }}=$ coeficiente convectivo de transferência de massa $\left(\mathrm{cm} \cdot \mathrm{s}^{-1}\right) ; \mathrm{C}^{\infty}(\mathrm{t})=$ concentração "bulk" na fase líquida externa à partícula $\left(\mathrm{mg} \cdot \mathrm{cm}^{-3}\right)$.

Dois modelos foram testados para a isoterma de adsorção:

$$
\text { Freundlich: } \quad \mathrm{q}_{\mathrm{i}}=\mathrm{k} \mathrm{C}_{\mathrm{i}}^{\mathrm{n}}
$$

Com $\mathrm{k}$ e $\mathrm{n}=$ constantes do modelo a serem ajustados aos dados experimentais.

Langmuir:

$$
q_{i}=\frac{a_{0} b c_{i}}{1+b c_{i}}
$$

Com $\mathrm{q}_{0}=$ quantidade máxima adsorvida na fase sólida; $1 / \mathrm{b}=$ constante de equilíbrio; sendo $\mathrm{q}_{0} \mathrm{e}$ b também ajustados aos dados experimentais.

Como os dados experimentais de concentração ao longo do tempo são obtidos como uma média na fase líquida fora da partícula, os resultados do modelo devem ser expressos segundo uma concentração média calculada a partir da integração dos perfis de concentração no interior da partícula.

Para modelo de Freundlich: $\overline{\mathrm{C}}(\mathrm{t})=\mathrm{C}_{0}^{\infty}-\frac{3}{\mathrm{R}^{3}} \int_{0}^{\mathrm{R}}\left(\varepsilon_{\mathrm{P}}+\rho_{\mathrm{d}} \mathrm{knC} \mathrm{C}_{\mathrm{i}}^{\mathrm{n}-1}(\mathrm{t})\right) \mathrm{C}_{\mathrm{i}}(\mathrm{t}) \mathrm{r}^{2} \mathrm{dr}$

Para o modelo de Langmuir:

$$
\bar{C}(t)=C_{0}^{\infty}-\frac{3}{R^{3}} \int_{0}^{R}\left(\varepsilon_{P}+\frac{q_{0} b}{\left(1+b C_{i}(t)\right)^{2}}\right) C_{i}(t) r^{2} d r
$$

Onde: $\mathrm{C}_{0}^{\infty}$ é a concentração bulk na fase externa à partícula no tempo $\mathrm{t}=0$.

O número adimensional de Biot de massa é definido por: $\quad \mathrm{Bi}=\frac{\mathrm{K}_{\text {conv }} \mathrm{R}}{\text { Def }}$

As equações e condições que compõem o modelo foram resolvidas, no software Mathematica versão $5.1^{\circledR}$, pelo método de diferenças finitas, observando-se critérios de unicidade de solução, 
convergência numérica e fechamento de balanço de massa.

\subsection{Planejamento experimental}

O planejamento experimental é uma ferramenta utilizada em muitos processos de análise, formulações de novos sistemas de operações e aprimoramento de sistemas de operações usuais. $\mathrm{Na}$ obtenção na adsorção do herbicida Diuron na pinha foi avaliada a influência das seguintes variáveis: A) Tempo; B) Massa de pinha e C) Concentração inicial de Diuron; com seus respectivos níveis de variação mostrados na Tabela 1. Foi adotado um planejamento e delineamento central composto rotacional (DCCR).

Tabela 1 - Fatores e níveis estudados no planejamento experimental.

\begin{tabular}{|l|c|c|c|c|c|}
\hline \multirow{2}{*}{} & \multicolumn{5}{|c|}{ Níveis codificados } \\
\cline { 2 - 6 } & $-1,68$ & -1 & 0 & +1 & $+1,68$ \\
\hline Tempo (min) & 20 & 50 & 95 & 140 & 170 \\
\hline Massa de Pinha(g) & 1,0 & 2,0 & 3,5 & 5,0 & 6,0 \\
\hline Concentração Inicial de Diuron (g.L L $\left.^{-1}\right)$ & 0,3 & 1,0 & 2,0 & 3,0 & 3,7 \\
\hline
\end{tabular}

Os resultados obtidos foram analisados através do software Statistica ${ }^{\circledR}$ utilizando a Metodologia de Superfícies de Resposta (MSR) com o objetivo de obter a condição ótima de operação.

Para este teste estatístico, foi escolhido o modelo linear com os efeitos principais e suas interações. Também, foi suposto que os desvios variam segundo uma distribuição normal, sendo que, para o planejamento fatorial completo a média populacional $\eta\left(x_{1}, x_{2}\right)$ pode ser representada por uma combinação linear das variáveis x1 e x2 (Equação 7).

$$
\eta\left(x_{1}, x_{2}\right)=\beta_{o}++\beta_{1} x_{1}++\beta_{2} x_{2}
$$

Onde: $\beta_{0}$ é o valor da média global das respostas e $\beta_{1}$ e $\beta_{2}$ representam os valores populacionais dos efeitos lineares dos efeitos principais e do efeito da interação, por unidade de $\mathrm{x}_{1}$ e $\mathrm{x}_{2}$.

\section{RESULTADOS E DISCUSSÃO}

\subsection{Isotermas e Cinética de Adsorção}

Na Tabela 2 são apresentados os valores obtidos modelando os dados experimentais em batelada da adsorção do herbicida Diuron utilizando como meio adsorvente a pinha do Pinus elliotti. A isoterma e os ensaios cinéticos foram procedidos à temperatura de $20^{\circ} \mathrm{C}$.

Na Tabela 2 são apresentados os parâmetros ajustados para o modelo de isoterma de Freundlich, o qual apresentou melhor ajuste. $\mathrm{O}$ valor do número de Biot baixo significa que maior foi à influência da resistência externa sobre o mecanismo de difusão. 


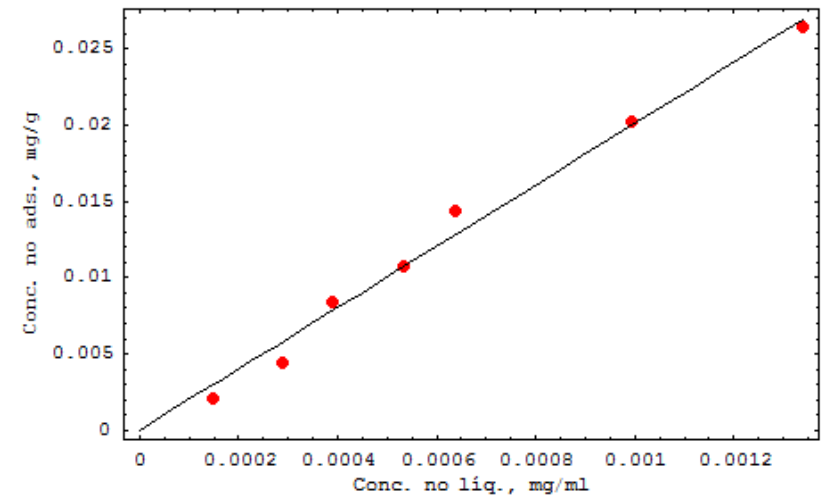

Figura 1: Isoterma de Adsorção do herbicida

Diuron em pinha- Modelo de Freundlich.

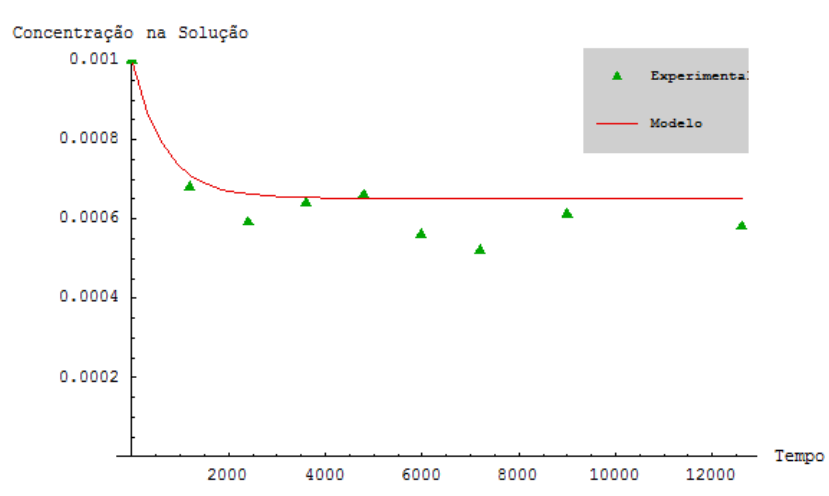

Figura 2: Cinética de Adsorção do Diuron em pinha $\left(\mathrm{C}_{0}=0,001 \mathrm{mg} / \mathrm{mL}\right)$.
Tabela 2 - Condições experimentais e parâmetros do modelo para o processo de adsorção em batelada.

\begin{tabular}{|c|c|}
\hline $\mathrm{R}(\mathrm{cm})$ & 0,313 \\
\hline $\mathrm{D}_{\mathrm{ef}}\left(\mathrm{cm}^{2} \min ^{-1}\right)$ & 0,0091 \\
\hline $\mathrm{K}_{\mathrm{conv}}\left(\mathrm{cm} \mathrm{\operatorname {min } ^ { - 1 } )}\right.$ & 0,00055 \\
\hline$\varepsilon_{\mathrm{p}}$ & 0,30 \\
\hline$\rho_{\mathrm{d}}\left(\mathrm{g} \mathrm{cm}^{-3}\right)$ & 0,25 \\
\hline $\begin{array}{l}\text { Conc. inicial de corante no } \\
\text { meio externo da partícula } \\
\qquad\left(\mathrm{mg} \mathrm{mL}^{-1}\right)\end{array}$ & 0,001 \\
\hline $\begin{array}{l}\text { Conc. de inicial de corante no } \\
\text { interior da partícula } \\
\left(\mathrm{mg} \mathrm{mL}^{-1}\right)^{*}\end{array}$ & 0,000001 \\
\hline Volume Solução (mL) & 40 \\
\hline Massa Adsorvente (g) & 1,0 \\
\hline Biot & 0,018 \\
\hline \multicolumn{2}{|l|}{$\begin{array}{l}\text { Parâmetros do modelo } \\
\text { Freundlich e índices } \\
\text { estatisticos }\end{array}$} \\
\hline $\mathrm{k}$ & 20,0449 \\
\hline $\mathrm{n}$ & 0,9993 \\
\hline Variança & $1,16 \times 10^{-6}$ \\
\hline Coef. de correlação & 0,9939 \\
\hline \multicolumn{2}{|c|}{$\begin{array}{l}\text { * Considerou-se uma pequena concentraçã } \\
\text { inicial na partícula devido à singularidad } \\
\text { apresentada pelo modelo de Freundlich en } \\
\text { concentração zero. }\end{array}$} \\
\hline
\end{tabular}

\subsection{Planejamento experimental}

Um planejamento estrela foi utilizado para avaliar a influência das variáveis: A) Tempo, B) Massa de adsorvente (Pinha) e C) Concentração inicial de herbicida (Diuron), com níveis codificados predefinidos, sobre a concentração final de Diuron no final do processo de adsorção. A Tabela 3 mostra a matriz de planejamento estrela e a as respostas obtidas para cada ensaio.

Através dos resultados obtidos na Tabela 3, realizou-se uma análise dos efeitos das três varáveis independentes estudadas sobre as respostas obtidas nos experimentos e seus respectivos índices estatísticos, que é apresentada na Tabela 4. 
Tabela 3 - Matriz do planejamento experimental estrela, com suas respectivas respostas.

\begin{tabular}{|c|c|c|c|c|}
\hline \multirow[t]{2}{*}{ Ensaio } & \multicolumn{3}{|c|}{ Fatores } & \multirow{2}{*}{$\begin{array}{c}\text { Resposta } \\
\text { Conc. final de Diuron } \\
\left(\mathrm{mg} . \mathrm{L}^{-1}\right)\end{array}$} \\
\hline & $\begin{array}{l}\text { Tempo } \\
(\min )\end{array}$ & $\begin{array}{l}\text { Massa de Pinha } \\
\text { (g) }\end{array}$ & $\begin{array}{l}\text { Conc. inicial de Diuron } \\
\left(\mathrm{mg} . \mathrm{L}^{-1}\right)\end{array}$ & \\
\hline 1 & $50(-1)$ & $2(-1)$ & $1(-1)$ & 0,283 \\
\hline 2 & $140(+1)$ & $2(-1)$ & $1(-1)$ & 0,02 \\
\hline 3 & $50(-1)$ & $5(+1)$ & $1(-1)$ & 0,234 \\
\hline 4 & $140(+1)$ & $5(+1)$ & $1(-1)$ & 0,017 \\
\hline 5 & $50(-1)$ & $2(-1)$ & $3(+1)$ & 0,668 \\
\hline 6 & $140(+1)$ & $2(-1)$ & $3(+1)$ & 0,07 \\
\hline 7 & $50(-1)$ & $5(+1)$ & $3(+1)$ & 0,066 \\
\hline 8 & $140(+1)$ & $5(+1)$ & $3(+1)$ & 0,033 \\
\hline 9 & $20(-1,68)$ & $3,5(0)$ & $2,0(0)$ & 0,516 \\
\hline 10 & $170(+1,68)$ & $3,5(0)$ & $2,0(0)$ & 0,433 \\
\hline 11 & $95(0)$ & $1,0(-1,68)$ & $2,0(0)$ & 0,659 \\
\hline 12 & $95(0)$ & $6,0(+1,68)$ & $2,0(0)$ & 0,327 \\
\hline 13 & $95(0)$ & $3,5(0)$ & $0,3(-1,68)$ & 0,108 \\
\hline 14 & $95(0)$ & $3,5(0)$ & $3,7(+1,68)$ & 1,443 \\
\hline 15 & $95(0)$ & $3,5(0)$ & $2,0(0)$ & 0,379 \\
\hline 16 & $95(0)$ & $3,5(0)$ & $2,0(0)$ & 0,404 \\
\hline 17 & $95(0)$ & $3,5(0)$ & $2,0(0)$ & 0,367 \\
\hline
\end{tabular}

$(-1,68),(-1),(0),(+1)$ e $(+1,68)$ são os Níveis de variação dos fatores.

Tabela 4- Cálculos dos efeitos e respectivos índices estatísticos em relação à resposta.

\begin{tabular}{|l|c|c|c|c|c|c|}
\hline & Efeito & $\begin{array}{c}\text { Desvio } \\
\text { Padrão }\end{array}$ & $\begin{array}{c}\text { Teste t } \\
\text { de } \\
\text { Student }\end{array}$ & Nível p & $\begin{array}{c}-95 \% \\
\text { Limite de } \\
\text { Confiança }\end{array}$ & $\begin{array}{c}+95 \% \\
\text { Limite de } \\
\text { Confiança }\end{array}$ \\
\hline Média/Interações & 0,3545 & 0,0046 & 77,4370 & 0,0002 & 0,3348 & 0,3742 \\
\hline (A) Tempo (min) & $-0,1833$ & 0,0102 & $-17,9329$ & 0,0031 & $-0,2273$ & $-0,1393$ \\
\hline $\begin{array}{l}\text { (B) Massa de pinha } \\
\text { (g) }\end{array}$ & $-0,1830$ & 0,0102 & $-17,9088$ & 0,0031 & $-0,2270$ & $-0,1391$ \\
\hline $\begin{array}{l}\text { (C) Conc. inicial de } \\
\text { Diuron (g.L-1) }\end{array}$ & 0,3702 & 0,0102 & 36,2232 & 0,0008 & 0,3262 & 0,4142 \\
\hline
\end{tabular}

Nota-se pela Tabela 4 que todos os efeitos são estatisticamente significativos para o nível de confiança adotado de $95 \%$, pois o fato do intervalo de confiança não conter o número zero indica que o fator em análise não apresenta efeito nulo, ou seja, ser considerado significativo no nível de 
confiança testado. Em relação à análise dos sinais algébricos dos efeitos encontrados, estes estão de acordo com o conhecimento que se tem dos fenômenos envolvidos. No que diz respeito à massa de pinha(B) um aumento da quantidade de adsorvente contribui para uma menor concentração do herbicida presente no final do processo de adsorção.

O modelo ajustado (Equação 8) é adequado para previsão de perfis do volume de gás produzido, conforme mostrado nas Figuras 2 a 4, que apresentam as curvas de nível geradas pelo modelo linear.
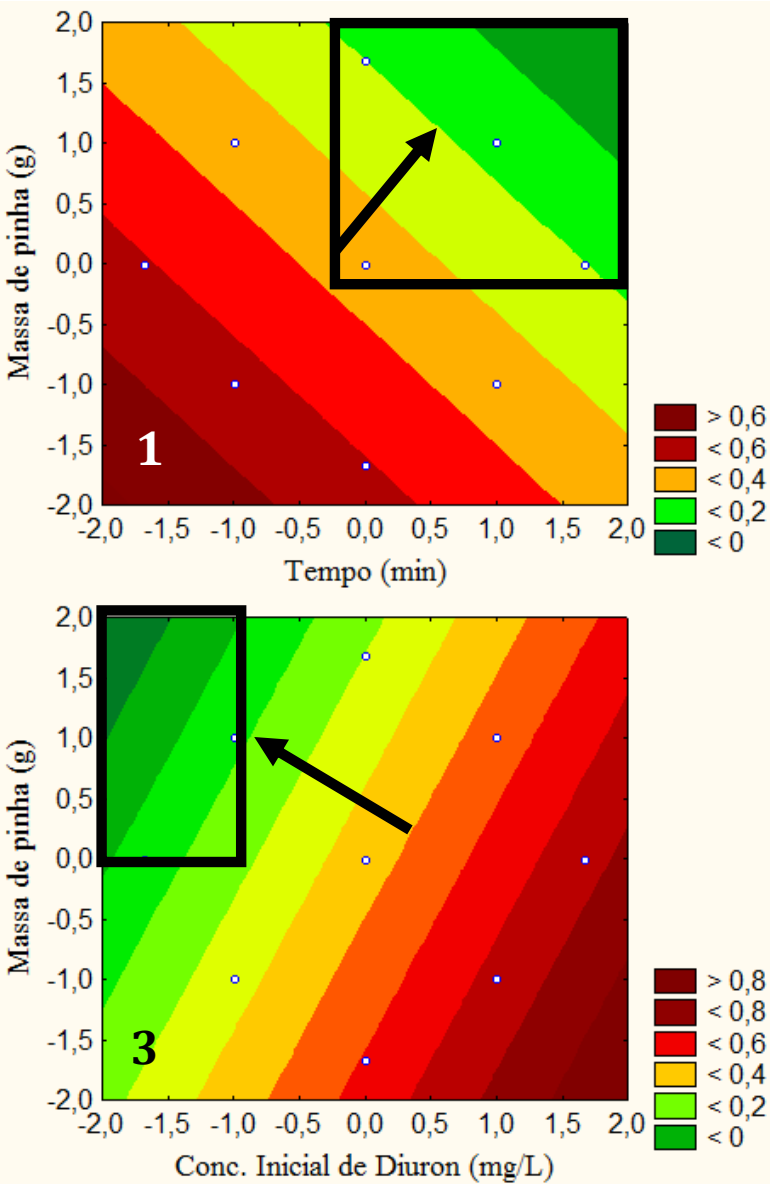

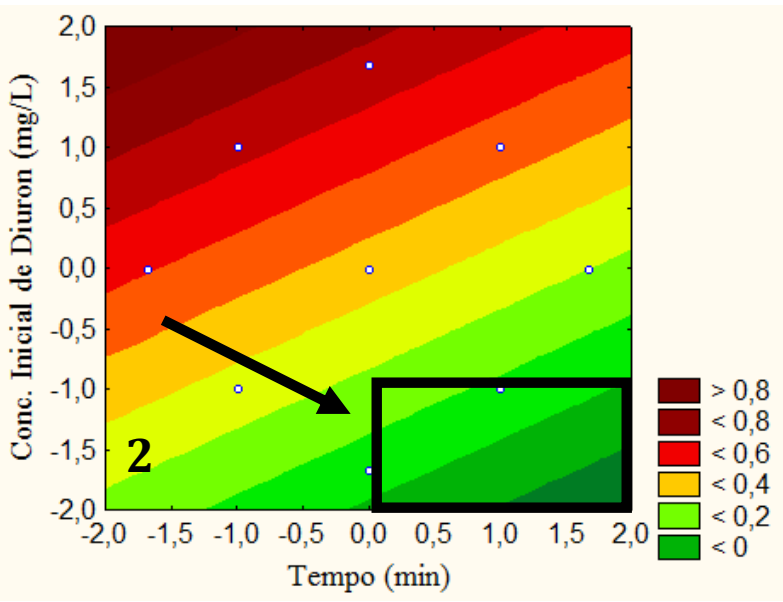

Figura 2: Gráfico de Curvas de níveis para os fatores Massa de Pinha e Tempo em relação à Concentração final de Diuron.

Figura 3: Gráfico de Curvas de níveis para os fatores Concentração inicial de Diuron e Tempo em relação à Concentração final de Diuron.

Figura 4: Gráfico de Curvas de níveis para os fatores Massa de Pinha e Concentração inicial de Diuron em relação à Concentração final de Diuron.

Conforme mostra as Figuras 2, 3 e 4 verifica-se, principalmente, que com o aumento da da massa de adsorvente, tempo e com a diminuição da concentração inicial de Diuron após o processo de adsorção, encontra-se num nível mínimo na região ótima de processo com massa de adsorvente de 3,5 a 6,5g (níveis 0 a +2,0), tempo de 95 a 185 minutos (níveis 0 a +2,0) e concentração inicial de Diuron abaixo de $1 \mathrm{mg} . \mathrm{L}^{-1}$ (nível -1). O modelo para o processo de adsorção do Diuron, para os níveis testados, é representado pela Equação 8.

$$
A=0,3545-0,0916 \mathrm{~B}-0,0915 \mathrm{C}+0,1851 \mathrm{D}
$$

onde: "A" é a concentração final de Diuron (g.L $\left.{ }^{-1}\right)$; B é o tempo (min); C é a massa de pinha (g); e D é a concentração inicial de Diuron (g.L $\left.\mathrm{L}^{-1}\right)$. 


\section{CONCLUSÕES}

Os resultados obtidos demonstram que a pinha apresenta um melhor ajuste para isoterma de Freundlich (coeficiente de correlação de 0,9939), todos os fatores testados se mostraram significativos e foi obtida uma adsorção máxima de $80 \%$ da concentração inicial de herbicida. Portanto, a pinha se mostrou um adsorvente promissor para a adsorção do Diuron em meio aquoso.

\section{REFERÊNCIAS}

MUSUMECI, M.P.; NAKAGAWA, L.E.; LUCHINI, L.C.; MATALLO, M.B.; ANDREA, M.M. Degradação do diuron-14C em solo e em plantas de cana-de-açúcar (Saccharum spp.). Pesq. Agro. Bras., 30, p.775-778, 1995.

NAMASIVAYAM, C. MINIASAMY, N.; GAYATRI, K.; RANI, M.; RANGANATHAN, K. Removal of dyes from aqueous solutions by cellulosic waste orange peel. Bioresour. Biotechnol., 57, p 37-43, 1996.

PORTO, R. L. L. Hidrologia Ambiental. Associação Brasileira de Recursos Hídricos, v. 3, 1991.

SINDAG - Sindicato Nacional das Indústrias de Defensivos Agrícolas, http://www.sindag.com.br. Acessada em: 23/04/2012.

SOARES, H. M. V Curso de Tratamento Biológico de Resíduos. Florianópolis: UFSC, cap. I.1. p. 11-12, 2005.

GONG, J.; YANG, C.; PU, W.; ZHANG, J. Liquid phase deposition of tungsten doped TiO2 films for visible light photoelectrocatalytic degradation of dodecylbenzenesulfonate, Chem. Eng. J. , 167, p. 190-197, 2011.

KATAYAMA, A.; MATSUMURA, F. Degradation of organochlorine pesticides, particularly endosulfan, by Trichoderma harzianus. Environ. Toxicol. Chem., 12, p. 1059-105, 1993.

AL-MUHTASE, A. H.; IBRAHIM, K. A.; ALBADARIN, A. B.; ALI-KASHMAN, O.; WALKER, G. M.; AHMAD, M. N. M. Remediation of phenol-contaminated water by adsorption using poly (methyl methacrylate) (PMMA), Chem. Eng. J. 168, p. 6991-699, 2011.

ZHOU, T; LIM, T.-T.; CHIN, S.-S.; FANE, A.G. Treatment of organics in reverse osmosis concentrate from a municipal wastewater reclamation plant: feasibility test of advanced oxidation processes with/without pretreatment, Chem. Eng. J., 166, p. 932-939, 2011. 